\title{
Investigation of the Benzene Molecule Adsorbed on Faujasite Zeolite Using Double Quantum Filtered NMR Spectral Analysis
}

\author{
Yu-Huei Chen and Lian-Pin Hwang* \\ Department of Chemistry, National Taiwan University, P.O. Box 23-34, Taipei, Taiwan, R.O.C., and Institute \\ of Atomic and Molecular Sciences, Academia Sinica, Taipei, Taiwan, R.O.C.
}

Received: August 11, 1998; In Final Form: March 24, 1999

\begin{abstract}
Deuteron NMR relaxation studies including double quantum filtered (DQF) spectral analyses, $\mathrm{T}_{1}$, and line widths are used to probe the dynamics and exchange process of various-loading benzene molecules adsorbed into NaY and dealuminated Y (DAY) including ultrastable Y (USY) zeolite. DQF NMR is a very sensitive method to determine the residual quadrupolar interaction resulting from the local order. In this work, we modified the cone model to describe the in-plane rotation around the hexad axis and the restricted wobbling motion of the benzene ring on the surface of the interaction site. The in-plane rotation and wobbling motion of adsorbed benzene molecules reflects a host-guest effect on the isolated adsorption site and also the mobility in the cage. In addition, the effect of a site-to-site hopping process results in a small net residual quadrupolar interaction. With increased loading, the in-plane rotation of benzene is significantly hindered in all faujasites but the population of adsorbed benzene dramatically reduces in DAY (including USY) systems only. This indicates the nature of site interaction on benzene molecules in a supercage. The population profile of adsorbate thus determined provides useful knowledge of loading effect, and the site interactions and distribution.
\end{abstract}

\section{Introduction}

A detailed knowledge of the interactions of adsorbate in zeolite systems is essential in understanding the adsorption and catalytic properties. Molecules with appropriate size and specific interaction such as benzene have been extensively used as a probe for the study of adsorption interaction and adsorbate dynamics. ${ }^{1-3}$ The benzene molecules in faujasite-type zeolites are preferentially adsorbed at cation sites which in the case of $\mathrm{NaY}$ are sodium cations located at site $\mathrm{S}_{\mathrm{II}}$ in the supercages. The less strong adsorption sites are the oxygen atoms of the 12-ring windows. ${ }^{4-6}$ In the case of dealuminated Y (DAY) including ultrastable Y (USY), benzene molecules are preferentially adsorbed at the hydroxyl groups. ${ }^{6}$ Evidence is known that benzene performs the rapid rotation about the sixfold symmetry axis and the hopping of benzene between the adsorption sites is associated with molecular reorientation. ${ }^{7-10}$ The behavior is consistent with results from molecular dynamic simulation and kinetic Monte Carlo calculation. ${ }^{1-13}$

A description of the theoretical background of NMR analysis of adsorption systems has been given by Pfeifer. ${ }^{7-9}$ Scharfer et al. employed two-dimensional (2D) exchange ${ }^{13} \mathrm{C}$ NMR techniques to the study of the reorientation dynamics of hydrocarbon molecules adsorbed on Ca-LSX zeolites with various loading. The study enabled the geometry and time scales of molecular hopping processes between adjacent adsorption sites to be characterized. ${ }^{14}$ Further, Isfort et al. used ${ }^{2} \mathrm{H}$ NMR exchange experiments to study the reorientational dynamics of benzene molecules at full occupancy of the supercages of $\mathrm{NaY}$. Experimental 2D spectra and 2D echo time-domain decay profiles are obtained and the values of the relevant correlation function can be measured directly. Comparison with model calculation gives evidence for tetrahedral-like jumps associated

* Corresponding author. Tel: +886-2-23668287. Fax: +886-2-23620200. E-mail: nmra@po.iams.sinica.edu.tw. with the small-angle "reorientation" motion for benzene molecules in the neighborhood. ${ }^{15}$ The small-angle reorientations of $\mathrm{C}_{6} \mathrm{D}_{6}$ are interpreted to be due to instantaneous relaxations after the hopping of neighboring molecules in the supercages. Furthermore, the small-angle jumps are about 2 times faster than the intersite jumps. The advantage of the exchange techniques and analyses is that certain results are unique and directly obtained without recourse to modeling.

Most NMR methods for studying adsorption report on only the $T_{1}$ and the usual line-width analysis. It can be difficult to analyze the adsorption effect due to site distribution in the supercage and the loading effects associated with intra- and intersupercage adsorption. However, Pearson et al. ${ }^{16}$ take advantages of intra- and intermolecular proton-proton dipolar interaction to generate multiple-quantum coherence in order to evaluate the spin cluster sizes of benzene and their distribution in various loading of $\mathrm{NaY}$ system. Furthermore, the recent developed multiquantum (MQ) MAS techenique has also been employed to examine the loading effect on ${ }^{23} \mathrm{Na}$ isotropic chemical shift and the second-order quadrupolar effects of sodium sites. ${ }^{17}$ The results show that with an increase in bulk benzene loading from 0 to 2 benzene molecules per supercage, there is an upfield trend in isotropic chemical shift and a decreasing second order quadrupolar effect for the $S_{\text {II }}$ sodium ions. With the loading from 2 to 5 benzene molecules per supercage, the variation of isotropic chemical shift and a secondorder quadrupolar effect are not significant.

The use of double quantum filtered (DQF) NMR spectroscopy ${ }^{18,19}$ as a diagnostic tool for the detection of anisotropy in macroscopically disordered systems has recently been developed. It may be utilized as a sensitive method for the determination of the residual quadrupolar interaction resulting from adsorbate dynamics among interaction sites in zeolite cages. In particular, for the $I=1$ spin system, the double quantum coherence vanishes in the isotropic medium, and hence 
Fast Motion Site

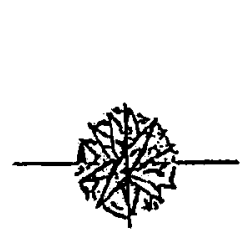

Exchange

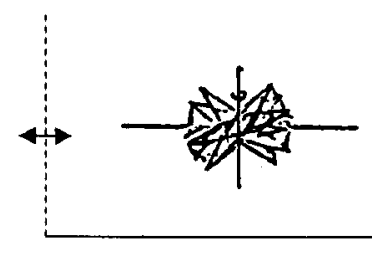

$\omega_{q}=0$

\section{Slow Motion Site}
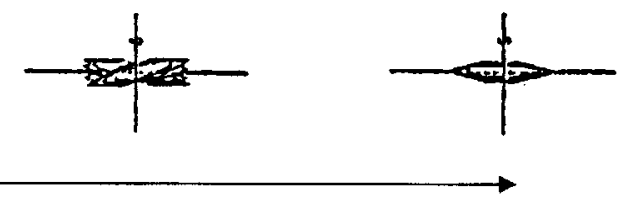

Adsorption

Coordinate $\omega_{q}=\omega_{q m}$

$S=1$

Figure 1. Schematic representation of benzene adsorption with exchange process in zeolite. The benzene molecule exchanges between the fast (f) and slow (s) sites. For slow sites, an adsorption coordinate is assumed to represent the various strengths of residual adsorption interaction $\left(\omega_{\mathrm{q}}\right)$ which is in turn related to the residual quadrupolar interaction and also to the order parameter $(S)$ which describes the degree of restriction in out-of-plane reorientation (the wobbling motion) for adsorbed benzene.

observation of the DQF system indicates the presence of the anisotropic motion of the spin-bearing molecules due to residual quadrupolar interaction. ${ }^{20}$ In the preliminary study on ultrastable Y (USY) and other faujasite zeolites, the observed usual deuteron spectra of benzene- $d_{6}$ retain the isotropic peak with no solid pattern over a broad temperature range. ${ }^{21-23}$ However, when observed in ${ }^{2} \mathrm{H}$ DQF spectra of $\mathrm{C}_{6} \mathrm{D}_{6}$ in USY, an additional central line appeared. ${ }^{23}$ This is evidence of the isotropic motion of the benzene through exchange with the benzene situated at adsorption sites with nonvanishing quadrupolar interaction.

For the quadrupolar system of adsorbate molecules, experiments show the collapse of the solid state pattern into a singlet at elevated temperatures. Based on this fact, it is assumed that the residual interaction on adsorption is averaged out by the isotropic motion among the various adsorption sites in the supercages. ${ }^{24,25}$ From a physical point of view, the adsorption sites in the supercages are not isotropically arranged in the supercages. Besides, it seems unreasonable to assume isotropic motion of the adsorbate molecule if it remains attached to the adsorption site for a significant time.

In this paper, we report on the use of deuteron NMR relaxation studies to investigate the dynamics and exchange process of benzene molecules adsorbed on faujasite-type zeolites, NaY, and dealuminated Y (DAY) including ultrastable Y (USY). Low loadings of zeolites with benzene are invoked to probe for site distribution and site interaction, and high loadings of zeolites with benzene for the sorbate-sorbate interaction in the same supercage of various faujasites. In addition to the usual investigation by $T_{1}$ and line-width analysis, DQF NMR with different creation times was also employed in the analyses. The order parameter pertinent to the model, the motional correlation times, and exchange parameters thus determined provide useful information on the adsorptive properties of the zeolite system.

\section{Formulation of Theory}

Two-Site Exchange Model with Residual Quadrupolar Interaction. The density matrix approach has been invoked in the study of exchange process with coherence transfer. The problem may be further simplified by expressing the rate equation on the basis of an irreducible tensorial $\operatorname{set}^{26,27}$ where the individual elements of the density matrix transform as components of the full rotation group. The element of $q$ th component of a rank $K$ irreducible tensor, $\sigma_{K q}$, can be obtained from the elements of the usual density matrix, $\rho_{m n}$, by

$$
\sigma_{K q} \equiv \sum_{m, n}(-1)^{m-1} \sqrt{2 K+1}\left(\begin{array}{lll}
I & I & \mathrm{~K} \\
\mathrm{~m} & -n & -q
\end{array}\right) \rho_{m n}
$$

where

$$
\left(\begin{array}{lll}
I & I & K \\
m & -n & -q
\end{array}\right)
$$

is a $3-j$ symbol $^{28}$ and $I$ is the nuclear spin quantum number of the relevant nuclei. The basic relaxation theory of these irreducible tensorial sets is the same as that used in previous work. ${ }^{29}$ Additionally, we have included adsorption effect and exchange process in the relaxation matrix $\mathbf{R}$. The equation of motion for this system defined in a rotating frame may be expressed by the following Liouville equationr

$$
\partial \sigma(t) / \partial t=(-i \mathrm{~L}+\mathbf{R}) \sigma(t)
$$

where $\sigma(t)$ is the density operator at time $t$. The components of $\sigma(t)$ are related to the irreducible tensorial components of interest. In eq 2 , we define $L \sigma(t) \equiv[\mathrm{H}, \sigma(t)]$, where $\mathrm{L}$ is the Liouville operator corresponding to the Hamiltonian of the residual quadrupolar interaction, $\mathrm{H}$, which is given by

$$
\begin{aligned}
H & \equiv \omega_{q, \theta}\left(I_{z}^{2}-I(I+1)\right) \\
& \equiv{ }_{1} \omega_{q}\left(3 \cos ^{2} \theta-1\right)\left(I_{z}^{2}-I(I+1)\right)
\end{aligned}
$$

where $I_{z}$ is the $z$ component of spin $I . \omega_{q, \theta}$ and $\omega_{q}$ are the residual quadrupolar interaction in the laboratory frame of reference and in the molecular frame, respectively. $\theta$ is the angle between the principal axis of the residual quadrupolar interaction in the molecular frame and the Zeeman field. Without exchange and spin relaxation processes, the equation of motion for the related irreducible tensorial component under the influence of residual quadrupolar interaction in a rotating frame is given by ${ }^{30}$

$$
\frac{\mathrm{d}}{\mathrm{d} t}\left[\begin{array}{l}
\sigma_{11} \\
\sigma_{21}
\end{array}\right]=\left[\begin{array}{cc}
0 & -i \omega_{q, \theta} \\
-i \omega_{q, \theta} & 0
\end{array}\right]\left[\begin{array}{l}
\sigma_{11} \\
\sigma_{21}
\end{array}\right]
$$

where $\sigma_{11} \equiv I_{+} / 2$ and $\sigma_{21} \equiv\left(I_{z} I_{+}+I_{+} I_{z}\right)$. The strength of interaction of the benzene molecule with the adsorption site depends on the distance between them and their relative orientation. For simplicity, it is reasonable to assume that the magnitude of $\omega_{q}$ increases with the adsorption "positions" close to an adsorption site. Thus, the variation of $\omega_{q}$ may be considered as the adsorption coordinate, and all positions of the adsorption coordinate are treated as the slow motion site for benzene molecules. The description of adsorption and dynamics along the adsorption coordinate is depicted in Figure 1. It should be noted that different types of faujasites possess various characteristic $\omega_{q}$. Besides, thermal and hopping averag- 

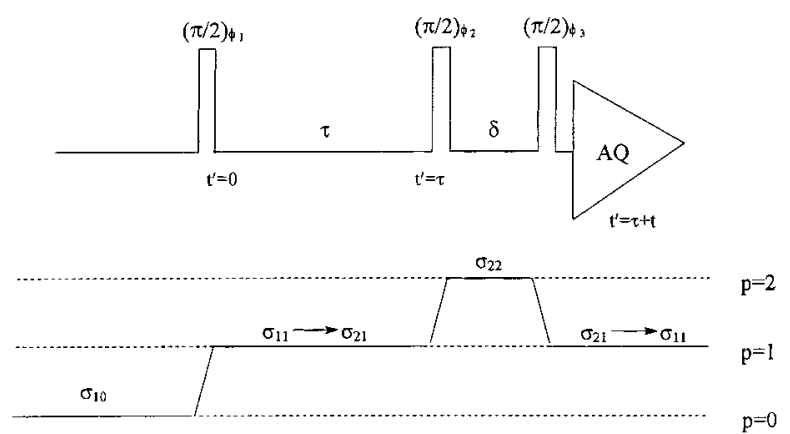

Figure 2. Pulse sequence and coherence-transfer pathway used in DQF experiment. $\sigma_{1 \mathrm{q}}$ represents the irreducible tensorial component with rank $l$ and coherence $q . \tau$ is the creation time for double quantum coherence and $\delta$ was set at $10 \mu \mathrm{s}$.

ing processes may render a smaller characteristic $\omega_{q}$. At room temperature and down to below $100 \mathrm{~K}$, the benzene molecules perform rapid internal rotation about their hexad axis in faujasite systems. ${ }^{9,11-13}$ Particularly, in NaY the intra- and intersupercage hopping between adsorption sites is associated with molecular reorientation. ${ }^{7-13}$ In order to treat the reorientational dynamics of benzene in faujasite systems, the relations among the motion, including internal rotation, wobbling motion, and site-to-site hopping motion, and the order parameter involved in a modified Brainard and Szabo's mode ${ }^{30,31}$ will be discussed below.

In DQF NMR, the detectable signal is generated from the second-rank tensor that is formed during the creation time of the double quantum filtered pulse sequence. The pulse sequence of the double quantum filtered NMR is shown in Figure 2. Since we have restricted our problem to the case of a spin 1 system as shown in eq 4, the only coherence-transfer pathways to be considered are between rank one and rank two alone. ${ }^{20,30}$ The first $\pi / 2$ pulse transforms the Zeeman order $\sigma_{10}\left(\sigma_{10} \equiv I_{z} / \sqrt{2}\right)$ into single-quantum coherence $\sigma_{11}$ which then generates $\sigma_{21}$ in the creation time, $\tau$, via the residual quadrupolar interaction given in eq 3 . The second $\pi / 2$ pulse with DQF phase cycling transforms $\sigma_{21}$ into double-quantum coherence $\sigma_{22}\left(\sigma_{22} \equiv I_{+} I_{+} /\right.$ 2 ) only. Since $\delta$ is set to be $10 \mu \mathrm{s}$, the relaxation of the $\sigma_{22}$ component may be neglected during time $\delta$. The last $\pi / 2$ pulse transforms the double-quantum coherence into an observable signal. In the present relaxation study, a representation of the molecular situation with exchange between a fast motion site $\mathrm{f}$ and a slow motion site $s$, characterized with a residual quadrupolar interaction, is needed to account for the adsorption process of benzene onto faujasites. The fast motion sites represent a dynamic state of benzene experiencing fast and highly symmetric reorientation. The slow motion site in $\mathrm{NaY}$ faujasite stands for the dynamic state of the benzene molecules in supercage affected by the $\mathrm{Na} \mathrm{S}_{\mathrm{II}}$ (and $\mathrm{W}$ ) sites interaction while for DAY and USY samples the dynamic state of the benzene molecules is rather affected by the hydroxyl aluminum group. It should be noted that $\omega_{q}$ is obtained after averaging over the coordinates of in-plane rotation, wobbling motion, and site-to-site hopping motion ${ }^{11,12}$ of the adsorbate molecule. Furthermore, the site-to-site hopping interaction is only considered in the case of multiple-site distribution such as in the adsorption cages of $\mathrm{NaY}$ faujasite. The benzene reorientation in slow motion site experiences a residual adsorption interaction, $\omega_{q}$. Hence, the evolution of related transverse components $\sigma_{11}$ and $\sigma_{21}$ during creation time may be augmented from eqs 2 and 4. It follows the rate equation of density operators: e.g.

$$
\frac{\mathrm{d}}{\mathrm{d} t}\left(\begin{array}{c}
\sigma_{11}^{\mathrm{f}} \\
\sigma_{21}^{\mathrm{f}} \\
\sigma_{11}^{\mathrm{s}} \\
\sigma_{21}^{\mathrm{s}}
\end{array}\right)=\left(\begin{array}{cccc}
-R_{11}^{\mathrm{f}}-k_{\mathrm{fs}} & 0 & k_{\mathrm{sf}} & 0 \\
0 & -R_{21}^{\mathrm{f}}-k_{\mathrm{fs}} & 0 & k_{\mathrm{sf}} \\
k_{\mathrm{fs}} & 0 & -R_{11}^{\mathrm{s}}-k_{\mathrm{sf}} & -i \omega_{q, \theta} \\
0 & k_{\mathrm{fs}} & -i \omega_{q, \theta} & -R_{21}^{\mathrm{s}}-k_{\mathrm{sf}}
\end{array}\right)
$$

where the residual quadrupolar interaction exists in s sites only, $k_{\mathrm{fs}}$ is the microscopic rate constant for transfer from site $\mathrm{f}$ to site $\mathrm{s}$, and $k_{\mathrm{sf}}$ is the microscopic rate constant for transfer from site $s$ to site $f$. In addition, the detailed balancing of the exchange process demands that

$$
P_{\mathrm{f}} k_{\mathrm{fs}}=P_{\mathrm{s}} k_{\mathrm{sf}}
$$

where we define $P_{\mathrm{f}}$ and $P_{\mathrm{s}}$ as the total populations in site $\mathrm{f}$ and site s, respectively. The inclusion of exchange process has placed not a limitation but rather a distribution suitable for studying the time scale of dynamics exchange process with adsorption confined inside the zeolite. In eq 5 , the spin relaxation rate constants for single and double quantum coherences (expressed by the first subscript of $R$ ) with different sites (expressed by the superscript) are given by ${ }^{30}$

$$
\begin{gathered}
R_{11}^{\mathrm{f}} \equiv C^{\mathrm{f}}\left(3 J_{0}^{\mathrm{f}}+5 J_{1}^{\mathrm{f}}+2 J_{2}^{\mathrm{f}}\right) \\
R_{21}^{\mathrm{f}} \equiv C^{\mathrm{f}}\left(3 J_{0}^{\mathrm{f}}+J_{1}^{\mathrm{f}}+2 J_{2}^{\mathrm{f}}\right) \\
R_{11}^{\mathrm{s}} \equiv C^{\mathrm{s}}\left(3 J_{0}^{\mathrm{s}}+5 J_{1}^{\mathrm{s}}+2 J_{2}^{\mathrm{s}}\right) \\
R_{21}^{\mathrm{s}} \equiv C^{\mathrm{s}}\left(3 J_{0}^{\mathrm{s}}+J_{1}^{\mathrm{s}}+2 J_{2}^{\mathrm{s}}\right)
\end{gathered}
$$

where we have $C^{\mathrm{f}} \equiv(3 / 160) \chi_{\mathrm{f}}^{2}$ and $C^{\mathrm{s}} \equiv(3 / 160) \chi_{\mathrm{s}}{ }^{2}$ and where $\chi_{\mathrm{f}}$ and $\chi_{\mathrm{s}}$ are defined as the quadrupolar coupling constants $\chi_{\mathrm{f}} /$ $2 \pi \equiv\left(e^{2} Q q_{\mathrm{f}} / h\right)$ and $\chi_{\mathrm{s}} / 2 \pi \equiv\left(e^{2} Q q_{\mathrm{s}} / h\right)$ for fast and slow motion sites, respectively. $J_{n}^{\mathrm{s}}$ and $J_{n}^{\mathrm{f}}$ are the spectral density functions, defined below for reorientational relaxation of benzene molecules in $\mathrm{f}$ and s sites, respectively. Since the $\mathrm{C}-\mathrm{D}$ bonding of the benzene molecule is considered to be cylindrical, the contribution of the asymmetry parameter $(\eta=0.06)$ of the electric field gradient to the relaxation interaction is negligible. ${ }^{33}$ It is worth noting that all the parameters used for a slow site have the characteristics of adsorption interactions. To guarantee the validity of this equation, the condition $\tau_{\mathrm{ex}} \gg \tau_{\mathrm{c}}$ must hold. Here $\tau_{\mathrm{c}}$ represents the microscopic motional correlation times related to the adsorbed benzene molecule and $\tau_{\mathrm{ex}}$ is defined as $\tau_{\mathrm{ex}} \equiv P_{\mathrm{s}} / k_{\mathrm{fs}}=P_{\mathrm{f}} / k_{\mathrm{sf}}$.

It is noted that eq 5 is invoked in the calculation of SQ and DQF spectra. In addition to the contribution from usual transverse spin relaxation, the residual quadrupolar interaction may also affect the line shapes of SQ and DQF spectra. The crucial feature of DQF spectra lies in the spectral lines with phase modulation resulting from the residual quadrupolar interaction. Furthermore, the strong residual quadrupolar interaction may affect dominantly in the shoulder of the spectral lines. For SQ spectra, it gives broadened shoulder while in DQF spectra it gives wiggling. The effect may be seen obviously in the DQF spectra of the systems with lower symmetric arranged adsorption sites, especially in the case of benzene in USY system. 
For the calculation of $T_{1}$ the time evolution of the longitudinal components follows the rate equation. ${ }^{29}$

$$
\frac{\mathrm{d}}{\mathrm{d} t}\left(\begin{array}{c}
\sigma_{10}^{\mathrm{f}} \\
\sigma_{10}^{\mathrm{s}}
\end{array}\right)=\left(\begin{array}{ll}
-R_{10}^{\mathrm{f}}-k_{\mathrm{fs}} & k_{\mathrm{sf}} \\
\mathrm{k}_{\mathrm{fs}} & -R_{10}^{\mathrm{s}}-k_{\mathrm{sf}}
\end{array}\right)\left(\begin{array}{c}
\sigma_{10}^{\mathrm{f}} \\
\sigma_{10}^{\mathrm{s}}
\end{array}\right)
$$

where $R_{10}^{\mathrm{f}}$ and $R_{10}^{\mathrm{s}}$ are the longitudinal relaxation matrix elements for site $\mathrm{f}$ and site $\mathrm{s}$, as defined by

$$
\begin{aligned}
& R_{10}^{\mathrm{f}} \equiv C^{\mathrm{f}}\left(J_{1}^{\mathrm{f}}+4 J_{2}^{\mathrm{f}}\right) \\
& R_{10}^{\mathrm{s}} \equiv C^{\mathrm{s}}\left(J_{1}^{\mathrm{s}}+4 J_{2}^{\mathrm{s}}\right)
\end{aligned}
$$

The calculations of $T_{1}$ values and the single quantum (SQ) line width were also employed in the determination of simulation parameters. The details of simulation are described in a later section on simulation.

Spectral Density for Benzene in Fast Motion Site. For a fast motion site, the reorientational Brownian motion of benzene molecules averages to zero the anisotropic quadrupole interaction experienced by the ${ }^{2} \mathrm{H}$ nucleus. The spectral density function expressed in eqs 7 and 9 for fast sites is given by ${ }^{34}$

$$
J_{n}^{\mathrm{f}}=\frac{3}{4} J_{n}^{\mathrm{fi}}+\frac{1}{4} J_{n}^{\mathrm{fo}}
$$

where $J_{n}^{\mathrm{fi}}$ and $J_{n}^{\mathrm{fo}}$ are the spectral density functions related to in-plane and out-of-plane rotations, respectively, and are given by

$$
\begin{aligned}
& J_{n}^{\mathrm{fi}}=\frac{2 \tau_{\mathrm{fi}}}{1+\left(n \omega_{0} \tau_{\mathrm{fi}}\right)^{2}} \\
& J_{n}^{\mathrm{fo}}=\frac{2 \tau_{\mathrm{fo}}}{1+\left(n \omega_{0} \tau_{\mathrm{fo}}\right)^{2}}
\end{aligned}
$$

where $\omega_{\mathrm{o}}$ is the Larmor frequency of deuteron nuclei and $\tau_{\mathrm{fi}}$ and $\tau_{\text {fo }}$ are the correlation times for in-plane rotation about the hexad axis and out-of-plane reorientational correlation times of the aromatic ring, respectively. For simplicity, in the present calculation, we use only one reorientational correlation time for benzene in a fast motion site; i.e., we let $\tau_{\mathrm{f}}=\tau_{\mathrm{fi}}=\tau_{\mathrm{fo}}$ in eqs 11 and 12 .

Spectral Density for Benzene in Slow Motion Sites. The spectral density functions for site $\mathrm{s}$ with consideration of residual quadrupolar interaction are defined by ${ }^{30}$

$$
\begin{aligned}
& J_{0}^{\mathrm{s}} \equiv j_{0}^{\mathrm{s}}\left(1+\frac{10}{7}\left\langle D_{00}^{(2)}\right\rangle+\frac{18}{7}\left\langle D_{00}^{(4)}\right\rangle-\left\langle D_{00}^{(2)}\right\rangle^{2}\right) \\
& J_{1}^{\mathrm{s}} \equiv j_{1}^{\mathrm{s}}\left(1+\frac{5}{7}\left\langle D_{00}^{(2)}\right\rangle-\frac{12}{7}\left\langle D_{00}^{(4)}\right\rangle\right) \\
& J_{2}^{\mathrm{s}} \equiv j_{0}^{\mathrm{s}}\left(1-\frac{10}{7}\left\langle D_{00}^{(2)}\right\rangle+\frac{3}{7}\left\langle D_{00}^{(4)}\right\rangle\right)
\end{aligned}
$$

where $j_{n}^{\mathrm{s}}$ is the spectral density, defined below, for the dynamic part of the motion at site $s, D_{00}^{(n)}$ are the Wigner rotation matrix elements ${ }^{28}$ with the orientation relative to the laboratory frame, and \langle\rangle means taking the average over space of motions not restricted by the adsorptive interactions, i.e., averaging over the coordinates of in-plane and wobbling motions. For multiple adsorption sites, additional averaging over the motion of siteto-site hopping is performed. In eq 13 the significance of the nonvanishing $\left\langle D_{00}^{(n)}\right\rangle$ represents the plateu term for orientation relaxation in the presence of residual quadrupolar interaction.

The model-free nonexponential function, such as Kohlrausch-Williams -Watts stretched exponential decay function, ${ }^{15}$ may be involved to evaluate the spectral density $j_{n}^{\mathrm{s}}$ for orientational relaxation. However, the dynamic behavior of the benzene molecules under the influence of adsorption is taken into account in the formulation of the time-correlation function. To describe the interference between the various modes of molecular motions in slow motion sites, we include in-plane rotation about the hexad axis of benzene, and wobbling motion of the ring normal uniformly in a cone. Therefore, it is convenient to modify the cone model of Brainard and Szabo ${ }^{31}$ to facilitate the calculation of the time-correlation function of the adsorbed benzene molecule. Note that the dynamic characteristics of various motional modes mentioned are presumably slower than the hopping process in order to introduce the coarse course graining-in-time approximation by means of averaging over the motions due to site-to-site hopping. Therefore, we leave out the overall reorientation of the whole molecule from the cone model due to adsorption onto zeolite. The spectral density function is defined by ${ }^{31,32}$

$$
\begin{aligned}
& j_{n}^{\mathrm{s}}=2\left\{\frac{3}{4} S^{2}\right. \frac{4 D_{\mathrm{i}}}{\left(4 D_{\mathrm{i}}\right)^{2}+\left(n \omega_{0}\right)^{2}}+ \\
& \frac{3}{4} \frac{\left(1-S^{2}\right)\left[4 D_{\mathrm{i}}+2 D_{\mathrm{w}} /\left(1-S^{2}\right)\right]}{\left[4 D_{\mathrm{i}}+2 D_{\mathrm{w}} /\left(1-S^{2}\right)\right]^{2}+\left(n \omega_{0}\right)^{2}}+ \\
&\left.\frac{1}{4} \frac{\left(1-S^{2}\right)\left[6 D_{\mathrm{w}} /\left(1-S^{2}\right)^{2}\right]}{\left[6 D_{\mathrm{w}} /\left(1-S^{2}\right)^{2}\right]^{2}+\left(n \omega_{0}\right)^{2}}\right\}
\end{aligned}
$$

It is assumed that the internal rotation and the wobbling motion of the adsorbed benzene ring have correlation times $\tau_{\mathrm{i}} \equiv 1 /\left(4 D_{\mathrm{i}}\right)$ and $\tau_{w} \equiv 1 /\left(6 D_{\mathrm{w}}\right)$, respectively. Here the wobbling motion is presumably slower than the in-plane rotation. These two motional correlation times are presumably larger than the reorientational correlation times $\tau_{\mathrm{fi}}$ and $\tau_{\text {fo }}$. The wobbling motion is assumed to be within a cone with the director normal to the adsorption site. The distribution of the cone may be expressed by an order parameter $S$ given by $S \equiv\left\langle D_{00}^{(2)}\right\rangle_{\mathrm{s}}$ where the average is over the in-plane rotation and wobbling motion defined in the frame $s$ of an adsorptive site. Here we have $S=0$ for completely random wobbling motion. For $S=1$, there is inplane rotation only and no wobbling motion. With this definition the second terms in eq 13 may be readily evaluated as $\left\langle D_{00}^{(2)}\right\rangle=$ $S D_{00}^{(2)}(\theta)$ where axial symmetry of the adsorption interaction and site distribution are assumed, while the third term in eq 13 gives $\left\langle D_{00}^{(4)}\right\rangle=\left\langle D_{00}^{(4)}\right\rangle_{\mathrm{s}} D_{00}^{(4)}(\theta)$. To evaluate the $\left\langle D_{00}^{(4)}\right\rangle_{\mathrm{s}}$ term in terms of $S$, a specific distribution of the cone model is needed. For isotropic distribution within a cone angle of $\beta$ with the director, Brainard and Szabo obtained $S=1 / 2 \cos \beta(1+\cos$ $\beta) .{ }^{30}$ We then obtain $\left\langle D_{00}^{(4)}\right\rangle_{\mathrm{s}}=(S / 8)\left(28 S-7(1+8 S)^{1 / 2}+1\right)$. The above formulation is for a simple adsorption in the supercage. For multiple sites in a supercage, \langle\rangle$_{\mathrm{s}}$ needs the additional average over the motions due to site-to-site hopping motion. Then $\theta$, as defined in eq 3 , may represent the angle between the principal axis of the averaged residual quadrupolar interaction in the molecular frame and the Zeeman field. Hence with the model given, one may evaluate the spectral density functions for site $s$. Furthermore, it should be noted that $\tau_{\mathrm{i}}$ and $\tau_{\mathrm{w}}$ are used in an averaged sense; i.e., their magnitudes remain 
the same irrespective of the variation of $S$ along the adsorption coordinates at a given temperature.

Residual Quadrupolar Interaction and Order Parameter in Adsorptive Process. The strength of adsorption interaction depends on the relative positions of the adsorbate and the adsorption site. The position with maximum $\omega_{\mathrm{q}}$, denoted by $\omega_{\mathrm{qm}}$, may be defined as the benzene molecule adsorbed on the adsorption site with maximum strength of interaction. At low temperature, the motion associated with $\omega_{\mathrm{qm}}$ involves in-plane motion around the hexad axis only, while there is neither wobbling motion of the ring normal nor site-to-site hopping motion. Thus, from both the experimental evidence and the symmetry arguments resulting from the fast internal motion around the hexad axis, the quadrupolar interaction of perdeuterobenzene molecules in this case reduces to half of the value obtained for rigid stationary benzene. Hence we take $\omega_{\mathrm{qm}}=$ $0.5 \omega_{\mathrm{q} 0}$ where $\omega_{\mathrm{q} 0} \equiv 3 / 4 \chi$. $^{9}$

Since both the $S$ and $\omega_{\mathrm{q}}$ parameters share the same degree of randomness by the same average over the motion associated with benzene, one may relate $S$ linearly with the residual quadrupolar anisotropy $\omega_{\mathrm{q}}$ with $S=\omega_{\mathrm{q}} / \omega_{\mathrm{qm}}$. The pictorial description of the order parameter and molecular motion along the adsorption coordinate is depicted in Figure 1. It should be noted that the adsorption process is of thermal kinetic origin and hence there exists a difference in adsorbate population along the adsorption coordinate as a consequence of exchange between fast and slow motion sites, and migration of adsorbate from different adsorption coordinates. Since exchange and migration are diffusive processes (see Appendix), a probability distribution of adsorbate along the adsorption coordinates may be represented approximately in an exponential form by

$$
f\left(\omega_{\mathrm{q}}\right)=\frac{\exp \left(-\omega_{\mathrm{q}} / \omega_{\mathrm{c}}\right)}{\omega_{\mathrm{c}}\left(1-\exp \left(-\omega_{\mathrm{qm}} / \omega_{\mathrm{c}}\right)\right)}
$$

where $\omega_{\mathrm{c}}$ characterizes a correlation strength of residual quadrupolar interaction. Beyond this value the population of adsorbate molecules with $\omega_{\mathrm{q}}>\omega_{\mathrm{c}}$ drops off significantly. Equation 15 is normalized in the range from 0 to $\omega_{\mathrm{qm}}$. The adsorbate distribution function given in eq 15 may be expressed in terms of the correlated order parameter $S_{\mathrm{c}} \equiv \omega_{\mathrm{c}} / \omega_{\mathrm{qm}}$. For faujasite, with merely a single site in the supercage, the average over the adsorption coordinates with the help of eq 15 represents the thermal kinetic origin of adsorption process mentioned previously. Since $\omega_{\mathrm{q}}$ is the result after averaging over all the motions concerned, the effective $\omega_{\mathrm{q}}$ represents an average over site-to-site hopping and the accompanying small-angle reorientations for multiple-site adsorption. ${ }^{11-13,15}$ Therefore, with the hopping process, one may thus expect a small effective residual quadrupolar interaction and thus a small $\omega_{\mathrm{c}}$ (or $S_{\mathrm{c}}$ ) value, in particular in the system of symmetrical averaged adsorption sites such as $\mathrm{Na} \mathrm{S}_{\text {II }}$ sites and $\mathrm{W}$ sites in NaY system. The use of eq 15 to describe the effect of multiple-site distribution in a supercage is only valid within the limit of fast site-to-site hopping; i.e., we have $\tau_{\text {hop }}{ }^{-1} \gg \omega_{\mathrm{c}}$, where $\tau_{\text {hop }}$ is the correlation time of the site-to-site hopping process.

Calculation of $\boldsymbol{T}_{1}$. Here we describe the use of the above theory in simulating the free-induction decay signal after the inversion recovery pulse sequence (i.e., $\pi-\tau^{\prime}-\pi / 2-$ Acq.). The total equilibrium state multipoles can be calculated from the corresponding density matrix elements as $\sigma_{10, \text { eq }} \equiv \gamma \hbar B_{0} I(I+$ 1) $/ 3 \sqrt{2} k T$, where $\gamma$ is gyromagnetic ratio, $k$ is the Boltzmann constant, and $B_{0}$ is the Zeeman field.

Since we have exchanging species, the individual multipoles must be appropriately population weighted. The effects of the $\pi$ pulse are then calculated, and thus immediately after the $\pi$ pulse and including population weighting state multipoles as the initial condition before evolution of $\tau^{\prime}$ we have $\sigma_{10}^{\mathrm{f}}(0)=$ $-p_{\mathrm{f}} \sigma_{10, \text { eq }}$ and $\sigma_{10}^{\mathrm{s}}(0)=-p_{\mathrm{s}} \sigma_{10, \mathrm{eq}}$.

For the calculation of $T_{1}$, eq 8 is used to follow the evolution of the longitudinal magnetization and the "motional" behavior of the spins during the delay time $\tau^{\prime}$. In eq $8, R_{10}^{\mathrm{s}}$ is calculated from eqs 9 with the help of eqs 13 and 14 according to the distribution of $\omega_{\mathrm{q}}$ and $\theta$ for the slow motion site, and $R_{10}^{\mathrm{f}}$ is calculated from eq 9 with the help of eqs $10-12$ for the fast motion site. The probability distribution in terms of $\omega_{\mathrm{q}}$ is described in eq 15. An isotropic distribution of $\theta$ is applied to simulate the situation of the random orientational distribution of zeolite crystals in the powder sample. Then the signal of longitudinal magnetization after the delay time $\tau^{\prime}$ can be evaluated as

$M_{\mathrm{z}}\left(\tau^{\prime}\right) \propto \int_{0}^{\omega_{\mathrm{qm}}} f\left(\omega_{\mathrm{q}}\right) \mathrm{d} \omega_{\mathrm{q}} \int_{0}^{\pi} \sin \theta \mathrm{d} \theta\left(\sigma_{10}^{\mathrm{f}}\left(\tau^{\prime}\right)+\sigma_{10}^{\mathrm{s}}\left(\tau^{\prime}\right)\right)$

The calculation is performed by numerical integration ${ }^{29}$ and the $T_{1}$ values can then be evaluated from $M_{\mathrm{z}}$ at various $\tau^{\prime}$.

Simulation of Double Quantum Spectra. We simulate the double quantum spectra in accordance with the DQF pulse sequence shown in Figure 2. The first $\pi / 2$ pulse transforms the Zeeman order $\sigma_{10 \text {,eq }}$ into single-quantum coherence $\sigma_{11}$ which then generates $\sigma_{21}$ in the creation time $\tau$ via the residual quadrupolar interaction given in eq 3 . Equation 5 is used to calculate the evolution of $\sigma_{11}^{\mathrm{f}}, \sigma_{21}^{\mathrm{f}}, \sigma_{11}^{\mathrm{s}}$, and $\sigma_{21}^{\mathrm{s}}$ during the creation time $\tau$ with the initial condition $\sigma_{11}^{\mathrm{f}}(0)=(1 / \sqrt{2}) P_{\mathrm{f}} \sigma_{10 \text {,eq }}$, $\sigma_{11}^{\mathrm{s}}(0)=(1 / \sqrt{2}) P_{\mathrm{s}} \sigma_{10, \mathrm{eq}}$, and $\sigma_{21}^{\mathrm{f}}(0)=\sigma_{21}^{\mathrm{s}}(0)=0$. In eq $5, R_{11}^{\mathrm{s}}$ and $R_{21}^{\mathrm{s}}$ are calculated from eqs 7 with the help of eqs 13 and 14 according to the distribution of $\omega_{\mathrm{q}}$ and $\theta$ for the slow motion site, and $R_{11}^{\mathrm{f}}$ and $R_{21}^{\mathrm{f}}$ are calculated from eqs 7 with the help of eqs $10-12$ for the fast motion site. In Figure 2, the signal immediately after the third $\pi / 2$ pulses with DQF phase cycling is proportional to the quantity of double quantum coherence $\sigma_{21}^{\mathrm{f}}(\tau)$ and $\sigma_{21}^{\mathrm{s}}(\tau)$. Thus with the initial condition $\sigma_{21}^{\mathrm{f}}=\sigma_{21}^{\mathrm{f}}(\tau)$, $\sigma_{21}^{\mathrm{s}}=\sigma_{21}^{\mathrm{s}}(\tau)$, and $\sigma_{11}^{\mathrm{f}}=\sigma_{11}^{\mathrm{s}}=0$ (see Figure 2), eq (5) is used again to calculate the the free induction decay (FID) during the acquisition time $t$. In analogy to eq 16, the FID signal for the transverse relaxation is proportional to

$$
\int_{0}^{\omega_{\mathrm{qm}}} f\left(\omega_{\mathrm{q}}\right) \mathrm{d} \omega_{\mathrm{q}} \int_{0}^{\pi} \sin \theta \mathrm{d} \theta\left(\sigma_{11}^{\mathrm{f}}(\tau+t)+\sigma_{11}^{\mathrm{s}}(\tau+t)\right)
$$

The DQF spectra may be obtained by the previously described Fourier transform procedure. ${ }^{28}$

Calculation of Line Width. Since the usual spectra were obtained by one-pulse experiment, the $\pi / 2$ pulse transforms the Zeeman order $\sigma_{10 \text {,eq }}$ into single-quantum coherence $\sigma_{11}$ with the same initial condition $\sigma_{11}^{\mathrm{f}}(0), \sigma_{11}^{\mathrm{s}}(0), \sigma_{21}^{\mathrm{f}}(0)$, and $\sigma_{21}^{\mathrm{s}}(0)$ defined in the previous section. Equation 5 is used to calculate the evolution of $\sigma_{11}^{\mathrm{f}}$ and $\sigma_{11}^{\mathrm{s}}$ during the acquisition time $t$. In analogy to eq 17, the usual spectrum may be obtained from the FID signal expressed by $\left(\sigma_{11}^{\mathrm{s}}(t)+\sigma_{11}^{\mathrm{f}}(t)\right)$. Then the full widths at half-height $\Delta v_{1 / 2}$ and one-third height were calculated.

\section{Experimental Section}

The NaY (LZY-52, Si/Al = 2.4) used was supplied and characterized by Union Carbide Co., USA. Two dealuminated Y samples (CBV720, $\mathrm{Si} / \mathrm{Al}=15$ and $\mathrm{CBV780}, \mathrm{Si} / \mathrm{Al}=40)$ used were supplied and characterized by PQ Zeolites, Holland. 
Hereafter we denote the former as DAY and the latter as USY. $\mathrm{N}_{2}$ adsorption-desorption isotherm for USY at $77 \mathrm{~K}$ was measured with Micrometric ASAP 2100. The adsorbate material, $\mathrm{C}_{6} \mathrm{D}_{6}$, was purchased from Merck. With a minimum purity of $99.5 \%$, no further purification process was used.

One small glass ball containing the required amount of $\mathrm{C}_{6} \mathrm{D}_{6}$ sample was sealed and then admitted to the upper part of the sample tube with glass wool separating it from the zeolite in the lower part of the tube. The sample was dehydrated under a $10^{-5}$ Torr vacuum at $673 \mathrm{~K}$ for at least $12 \mathrm{~h}$. After cooling, the tube was sealed, leaving the dried zeolite and the $\mathrm{C}_{6} \mathrm{D}_{6}$-carrying ball in the tube. The $\mathrm{C}_{6} \mathrm{D}_{6}$-carrying ball was then broken by shaking the tube. The lower part of the sample tube was chilled in liquid $\mathrm{N}_{2}$. The section of the NMR tube containing the loaded zeolite powder was sealed and separated from the upper part containing the rest of the glass capillary ball. Afterwards, the sample was then kept at $353 \mathrm{~K}$ for $12 \mathrm{~h}$ to ensure a homogeneous distribution of adsorbate onto the zeolite. Reproducibility of relaxation data was checked to examine whether equilibrium is reached in all supercages over a period of 4 months. The loadings of $\mathrm{C}_{6} \mathrm{D}_{6}$ (molecule per supercage) in various samples are as follows: for low-loading samples NaY 0.5, DAY 0.8; and for high-loading samples NaY 3.0, DAY 3.9, USY 3.2.

The ${ }^{2} \mathrm{H}$ NMR measurements were performed on Bruker MSL500 and MSL-300 spectrometers operating at $76.78 \mathrm{MHz}(11.75$ $\mathrm{T})$ and $46.07 \mathrm{MHz}(7.05 \mathrm{~T})$, respectively. In a quadrupolar echo measurement, a $\pi / 2$ pulse of $7 \mu \mathrm{s}$ and a delay of $10 \mu \mathrm{s}$ was used to obtain the solid spectrum of adsorbed benzene at 130 $\mathrm{K}$. For transverse relaxation studies, the line widths $\Delta v_{1 / 2}$ were measured as the full width at half-height from the one-pulse experiment. The magnetic inhomogeneity was estimated by comparing the Hahn spin-echo and line-width measurement of $\mathrm{C}_{6} \mathrm{D}_{6}$ liquid. The magnetic inhomogeneity was found to account for less than $5 \mathrm{~Hz}$ of the observed line width at half-height in the spectra. The longitudinal relaxation measurements were obtained using the inversion-recovery pulse sequence. The ${ }^{2} \mathrm{H}$ $T_{1}$ and line-width measurements were performed on Bruker MSL-500 and MSL-300 spectrometers operating at $76.78 \mathrm{MHz}$ $(11.75 \mathrm{~T})$ and $46.07 \mathrm{MHz}(7.05 \mathrm{~T})$, respectively, with $\pi / 2$ pulse length of about $15 \mu \mathrm{s}$. A delay of at least $5 T_{1}$ was allowed between scans for the $T_{1}$ and DQF spectra with proper phase cycling. DQF spectra are conventionally measured using the pulse sequence $\pi / 2-\tau^{\prime}-\pi / 2-\delta-\pi / 2-$ (acq.). Since there is no field-dependent effect, the DQF spectra were displayed at 76.78 $\mathrm{MHz}$ only. The temperature was controlled to within $\pm 0.1{ }^{\circ} \mathrm{C}$ and was calibrated using a methanol reference. There are errors of approximately $10 \%$ associated with $T_{1}$ and line-width measurements, respectively.

\section{Results}

To understand any possible change of structure after dealumination, the tetrahedral/octahedral Al ratios of DAY and USY, as determined by ${ }^{27} \mathrm{Al}$ MAS NMR, are obtained as 4.8 and 5.2, respectively. IR studies of dealuminated Y shows that hydroxyl aluminum groups on extraframework $\mathrm{Al}$ atoms are inaccessible to benzene molecules. ${ }^{6}$ Furthermore, in accordance with $\mathrm{N}_{2}$ adsorption-desorption isotherm experiment, the microporous/ mesoporous adsorption surface ratio for USY is 5.6 if spherical pores are assumed. Thus, one may expect a higher microporous/ mesoporous adsorption surface ratio for DAY system. Therefore, benzene molecules can be considered as being dominantly adsorbed on supercage but not on mesopores sites formed in DAY and USY.

In the present work, DQF spectra, line widths, and $T_{1}$ measured at 76.78 and $46.07 \mathrm{MHz}$ are obtained for samples
TABLE 1: Experimental and Calculated ${ }^{2} \mathrm{D} T_{1}$ and Line Width in Low-Loading Samples Measured at Various Temperatures and Two Resonance Fields ${ }^{a}$

\begin{tabular}{|c|c|c|c|c|c|c|c|c|c|}
\hline \multirow[b]{3}{*}{ zeolite } & \multirow{3}{*}{$\begin{array}{l}\text { temp } \\
(\mathrm{K})\end{array}$} & \multicolumn{4}{|c|}{$T_{1}(\mathrm{~ms})$} & \multicolumn{4}{|c|}{$\Delta v_{1 / 2}(\mathrm{~Hz})$} \\
\hline & & \multicolumn{2}{|c|}{$46.07 \mathrm{MHz}$} & \multicolumn{2}{|c|}{$76.78 \mathrm{MHz}$} & \multicolumn{2}{|c|}{$46.07 \mathrm{MHz}$} & \multicolumn{2}{|c|}{$76.78 \mathrm{MHz}$} \\
\hline & & calc & $\exp$ & calc & $\exp$ & calc & $\exp$ & calc & $\exp$ \\
\hline \multirow[t]{4}{*}{$\mathrm{NaY}$} & 290 & 44 & 48 & 121 & 117 & 4780 & 4100 & 4800 & 4332 \\
\hline & 310 & 27 & 30 & 73 & 68 & 2600 & 2630 & 2560 & 2532 \\
\hline & 330 & 17 & 21 & 46 & 42 & 1587 & 1625 & 1600 & 1523 \\
\hline & 350 & 14 & 18 & 37 & 30 & 1213 & 1265 & 1120 & 1159 \\
\hline \multirow[t]{4}{*}{ DAY } & 210 & 36 & 34 & 45 & 65 & 1320 & 1300 & 1280 & 1400 \\
\hline & 250 & 33 & 29 & 50 & 54 & 589 & 580 & 600 & 610 \\
\hline & 290 & 37 & 35 & 55 & 52 & 354 & 345 & 320 & 360 \\
\hline & 330 & 38 & 43 & 62 & 62 & 230 & 270 & 220 & 250 \\
\hline \multirow[t]{4}{*}{$\mathrm{USY}^{b}$} & 210 & 44 & 39 & 52 & 73 & 1580 & 1690 & 1525 & 1600 \\
\hline & 250 & 37 & 33 & 57 & 57 & 596 & 540 & 587 & 571 \\
\hline & 290 & 37 & 35 & 61 & 58 & 228 & 230 & 201 & 219 \\
\hline & 330 & 45 & 51 & 69 & 76 & 148 & 150 & 136 & 141 \\
\hline
\end{tabular}

${ }^{a} 10 \%$ error is expected in all values. ${ }^{b}$ This result has been shown in ref 16 .

TABLE 2: Experimental and Calculated ${ }^{2} D T_{1}$ and Line Width in High-Loading Samples Measured at Various Temperatures and Two Resonance Fields ${ }^{a}$

\begin{tabular}{|c|c|c|c|c|c|c|c|c|c|}
\hline \multirow[b]{3}{*}{ zeolite } & \multirow{3}{*}{$\begin{array}{c}\text { temp } \\
(\mathrm{K})\end{array}$} & \multicolumn{4}{|c|}{$T_{1}(\mathrm{~ms})$} & \multicolumn{4}{|c|}{$\Delta v_{1 / 2}(\mathrm{~Hz})$} \\
\hline & & \multicolumn{2}{|c|}{$46.07 \mathrm{MHz}$} & \multicolumn{2}{|c|}{$76.78 \mathrm{MHz}$} & \multicolumn{2}{|c|}{$46.07 \mathrm{MHz}$} & \multicolumn{2}{|c|}{$76.78 \mathrm{MHz}$} \\
\hline & & calc & $\exp$ & calc & $\exp$ & calc & $\exp$ & calc & $\exp$ \\
\hline \multirow[t]{3}{*}{$\mathrm{NaY}$} & 290 & 58 & 78 & 162 & 154 & 6220 & 6500 & 6222 & 6720 \\
\hline & 310 & 45 & 51 & 125 & 120 & 3000 & 3256 & 3111 & 3254 \\
\hline & 330 & 39 & 43 & 106 & 100 & 1757 & 1605 & 1700 & 1742 \\
\hline \multirow[t]{3}{*}{ DAY } & 210 & 28 & 27 & 35 & 37 & 400 & 378 & 414 & 400 \\
\hline & 250 & 45 & 46 & 65 & 62 & 185 & 165 & 177 & 182 \\
\hline & 290 & 63 & 68 & 104 & 98 & 144 & 140 & 148 & 148 \\
\hline \multirow[t]{3}{*}{ USY } & 210 & 40 & 37 & 45 & 48 & 264 & 256 & 260 & 264 \\
\hline & 250 & 58 & 54 & 75 & 72 & 156 & 158 & 150 & 150 \\
\hline & 290 & 76 & 78 & 120 & 114 & 130 & 140 & 133 & 132 \\
\hline
\end{tabular}

${ }^{a} 10 \%$ error is expected in all values.

described in the Experimental Section. For the purpose of comparison, data for the low-loading ( 0.5 benzene/supercage) USY sample presented previously are listed again in Table 1.23

The Value of $\omega_{\mathrm{qm}}$. We observe a powder pattern in the ${ }^{2} \mathrm{H}$ NMR spectrum for low-loading zeolite $\mathrm{NaY}$ at $130 \mathrm{~K}$. The edge splitting $\Delta v$ of the $\mathrm{NaY}$ sample is $69 \mathrm{kHz}$ which implies a quadrupolar coupling constant $\chi / 2 \pi$ of $184 \mathrm{kHz}$. This result agrees with that of Pfeifer ${ }^{9}$ and Sousa Goncalves et al. ${ }^{22}$ and suggests that immobile benzene is undergoing only in-plane rotation. However, a solid pattern with a central peak appeared in ${ }^{2} \mathrm{H}$ NMR spectra for the USY sample measured at 135 and $150 \mathrm{~K}^{23}$ The appearance of a central peak may suggest that a slow exchange process still exists at these temperatures. From these two measurements the same $67 \mathrm{kHz}$ edge splittings in USY sample are obtained. According to the above results, we may take an average value $\omega_{\mathrm{qm}}=68 \mathrm{kHz}$ or $\chi_{\mathrm{s}} / 2 \pi=181 \mathrm{kHz}$ as the maximum value $\omega_{\mathrm{qm}}$ in our calculation for all of the faujasite samples. Moreover, the quadrupolar coupling constant at the fast site may be estimated for $\mathrm{C}_{6} \mathrm{D}_{6}$ in liquid crystal phase. $\chi_{\mathrm{f}} / 2 \pi$ is estimated to be $183 \mathrm{kHz}$. Therefore, one may take $\chi_{\mathrm{f}} /$ $2 \pi \approx \chi_{\mathrm{s}} / 2 \pi=181 \mathrm{kHz}$ in our calculation.

Parameters and the Simulated DQF Spectrum. It is found that the simulated DQF spectrum is not sensitive to the variation $\tau_{\mathrm{i}}$ provided $\tau_{\mathrm{i}}<\tau_{\mathrm{w}} . P_{\mathrm{s}}, S_{\mathrm{c}}, \tau_{\mathrm{w}}$, and $k_{\mathrm{fs}}$ may be estimated from the line-width analysis and DQF spectrum with various DQ creation time $\tau$. In Figure 3, the effects of various parameters on spectral lines are demonstrated. Figure 3a shows the typical DQF spectra with the effects of the presence of fast motion site which result in a central peak with $P_{\mathrm{s}}$ dependent intensity 


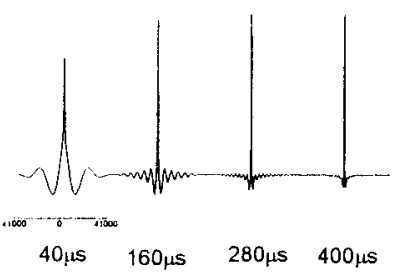

(a)
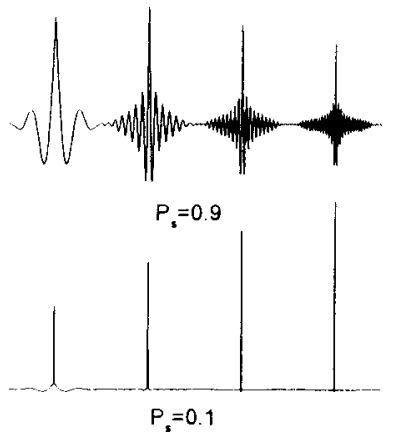

(c)
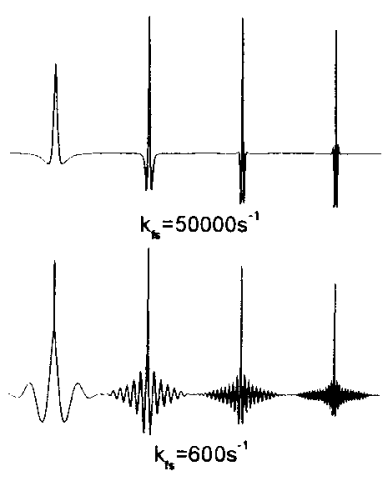

(e)

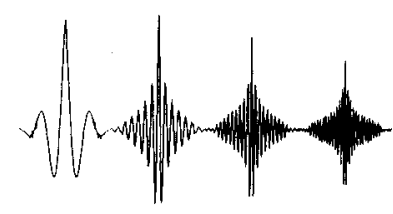

(b)
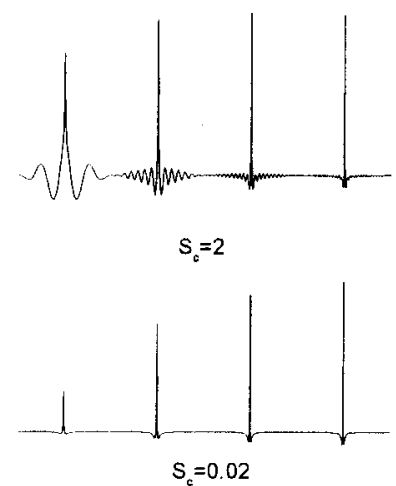

(d)
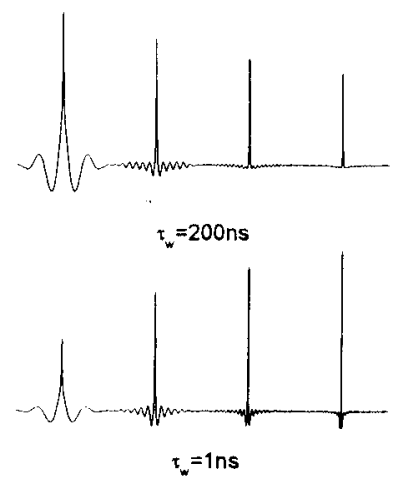

(f)
Figure 3. Effects of $P_{\mathrm{s}}, S_{\mathrm{c}}, k_{\mathrm{fs}}$, and $\tau_{\mathrm{w}}$ parameters on the simulated DQF spectra. The DQF spectra are not sensitive to $\tau_{\mathrm{i}}$ and $\tau_{\mathrm{f}}$ parameter provided that $\tau_{\mathrm{i}}<\tau_{\mathrm{w}}$ and $\tau_{\mathrm{f}}<10^{-10} \mathrm{~s}$. In (a) the parameters used are $P_{\mathrm{s}}=0.5, S_{\mathrm{c}}=0.7, k_{\mathrm{fs}}=3000 \mathrm{~s}^{-1}$, and $\tau_{\mathrm{w}}=50 \mathrm{~ns}$. In (b), the same parameters are used except $P_{\mathrm{s}}=1.0$. In (c) $-(\mathrm{f})$, one of the parameters used in (a) is altered and denoted in the buttom of the spectra.

(Figure 3c). However, it vanishes in the DQF spectra with no fast motion site considered (Figure $3 \mathrm{~b}, P_{\mathrm{s}}=1$ ).

As displayed in Figure 3, d and e, the wiggling wing in the DQF spectra disappears in the cases of $S_{\mathrm{c}} \ll 1$ and $k_{\mathrm{fs}} \tau \geq 1(\tau$ $=40 \mu \mathrm{s})$, respectively. A small $S_{\mathrm{c}}$ indicates that the effective residual quadrupolar interaction is insignificant. A large $k_{\mathrm{fs}}$ values represents a fast exchange process which accounts for the vanish of the wiggling wing due to the contribution from the fast motion site. Moreover, in Figure $3 \mathrm{e}$ as the creation times increase, the two patterns of negative intensity lines corresponding to $k_{\mathrm{fs}} \tau<1\left(k_{\mathrm{fs}}=600 \mathrm{~s}^{-1}\right)$ and $k_{\mathrm{fs}} \tau>1\left(k_{\mathrm{fs}}=50000 \mathrm{~s}^{-1}\right)$, respectively, display opposite trends as depicted in Figure 3e. Also in the case of $k_{\mathrm{fs}} \tau>1$, an intense negative peak is observed with increasing $k_{\mathrm{fs}} \tau$. Therefore, these effects may be used to distinguish the source for the vanishing of the wiggling wing.

The effect of wobbling motion may be revealed from the pattern of the central peaks with increases of creation time. As shown in Figure 3a,f, for long $\tau_{\mathrm{w}}$ it displays monotonically decreasing central peaks while for short $\tau_{\mathrm{w}}$ it shows a reverse trend with increasing creation time.
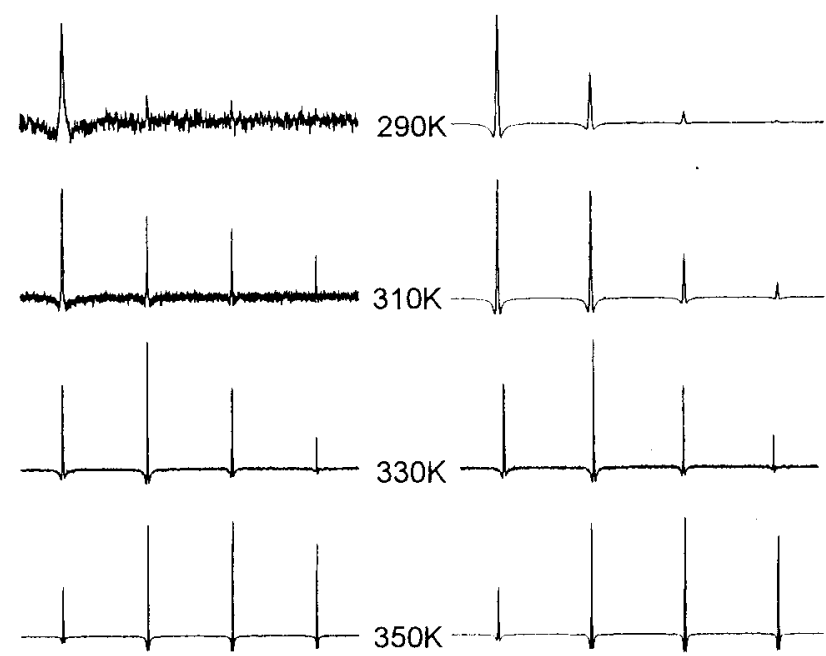

$.00000 \overline{0} 60000$

$\mathrm{Hz}$

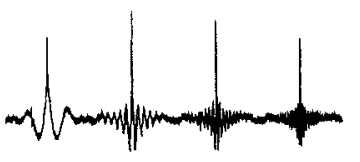

(a)
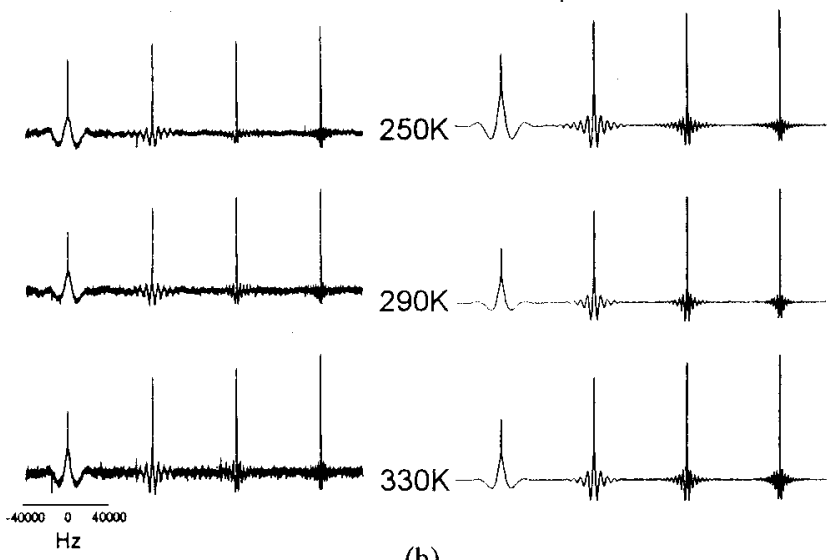

(b)

Figure 4. Experimental (left) and simulated (right) ${ }^{2} \mathrm{H}$ double quantum filtered spectra of (a) NaY and (b) DAY low-loading samples at the various cited temperatures. For a given temperature, the four DQF spectra from left to right are obtained with DQ creation time $t$ given by $40,160,280$, and $400 \mu$ s, respectively.

Estimation of $\boldsymbol{\tau}_{\mathbf{f}}$ from DQF Spectra. Under the exchange process, the contribution of the fast motion site results in the central peak which appears in Figures 4 and 5 for USY and DAY samples. For NaY samples, the central peak is not so obvious especially for a small population of the fast motion site $\left(P_{\mathrm{f}} \sim 0.1\right)$. Thus, all the simulated results are insensitive to $\tau_{\mathrm{f}}$ as long as the magnitudes are kept below $10^{-10} \mathrm{~s}$. The motional narrowing conditions are required for $\tau_{\mathrm{f}}$ in order to match the narrow width of the central peak.

Parameter Fitting Algorithm. Except the one observed at $350 \mathrm{~K}$ in $\mathrm{NaY}$ system showing almost equally negative intensity with increasing creation time $\tau$ (Figure 4a), all the intensity of negative peaks of experimental DQF spectra decreases as the creation time increases from $\tau=160 \mu \mathrm{s}$. Following the simulated DQF spectra with various $k_{\mathrm{fs}}, P_{\mathrm{f}}$, and $S_{\mathrm{c}}$, the upper bound of $k_{\mathrm{fs}}$ may be set to be $10^{5} \mathrm{~s}^{-1}$ since a large $k_{\mathrm{fs}}$ yields a DQF spectra similar to the spectra shown in Figure $3 \mathrm{e}$ with $k_{\mathrm{fs}}$ $=50000 \mathrm{~s}^{-1}$. Nevertheless, the appropriate $k_{\mathrm{fs}}$ value for the DQF spectra at $350 \mathrm{~K}$ in $\mathrm{NaY}$ system should be about the maximum value we set. 


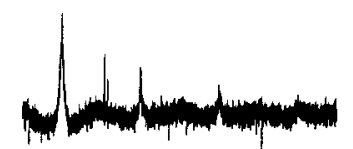

the
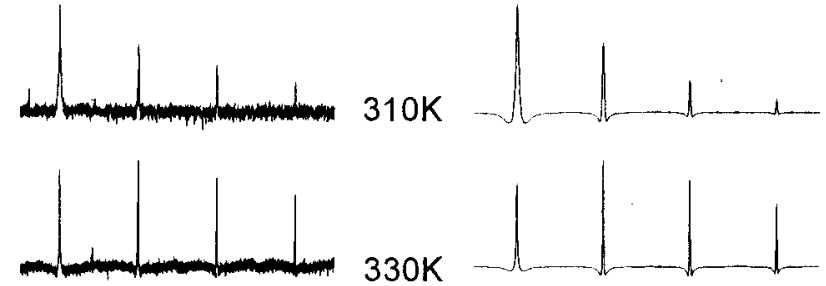

3
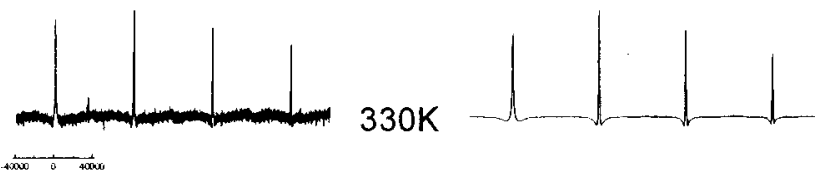

$\mathrm{Hz}$

(a)
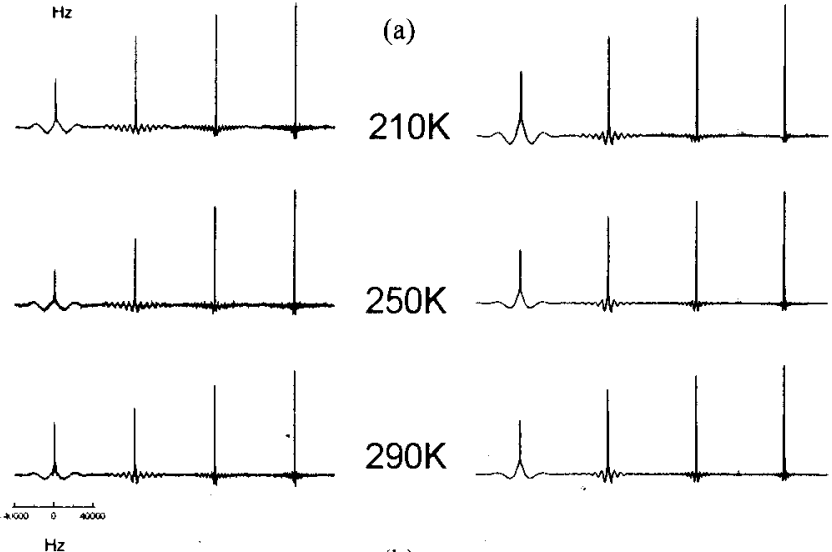

$\mathrm{Hz}$

(b)
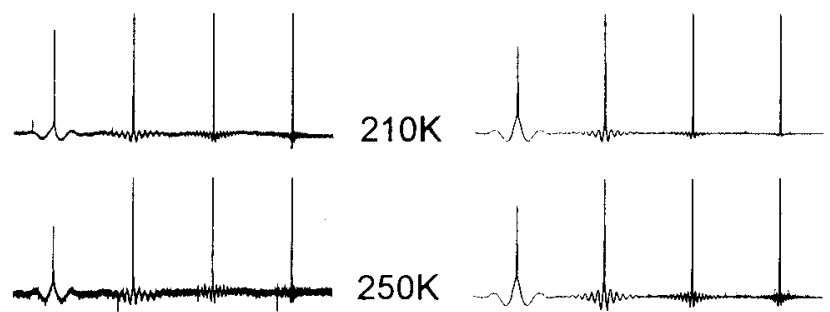

$250 \mathrm{~K}$
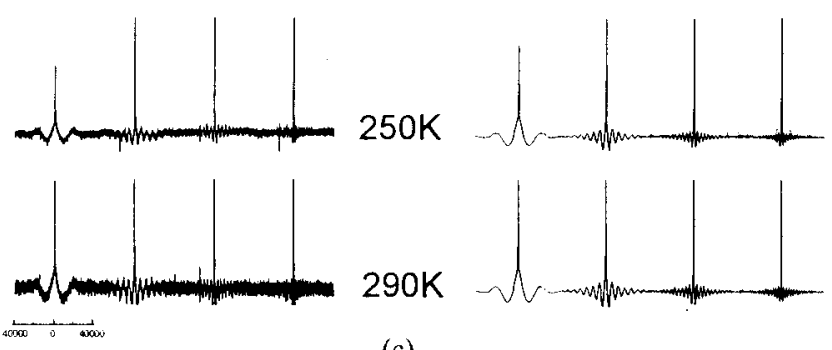

$\mathrm{Hz}$

$290 \mathrm{~K}$

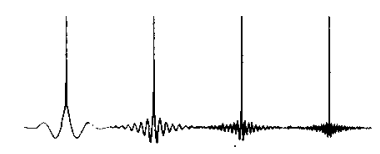

(c)

Figure 5. Experimental (left) and simulated (right) ${ }^{2} \mathrm{H}$ double quantum filtered spectra of (a) NaY, (b) DAY, and (c) USY high-loading samples at the various cited temperatures. For a given temperature, the four DQF spectra from left to right are obtained with DQ creation time $t$ given by $40,160,280$, and $400 \mu$ s, respectively.

As we examine the DQF spectra of NaY system at $290 \mathrm{~K}$, the effective $S_{\mathrm{c}}$ is insignificant and the contribution to the spectra is dominantly from the slow motion site. The upper limit of $\tau_{\mathrm{w}}$ may be estimated from the line width obtained in NaY system at this temperature; i.e., in accordance with eqs 5 and 7 the upper bound of $\tau_{\mathrm{w}}$ may be estimated from the line width $\Delta v_{1 / 2}$ $=(1 / \pi) R_{11}^{\mathrm{s}} \approx(3 / 2 \pi) C^{\mathrm{s}} \tau_{\mathrm{w}}$. It yields $\tau_{\mathrm{w}} \approx 500 \mathrm{~ns}$. The lower bound of $\tau_{\mathrm{w}}$ may be determined from the $\omega_{0} \tau_{\mathrm{w}} \gg 1$ in accordance with field-independent single quantum and DQF spectra. It yields $\tau_{\mathrm{w}} \approx 3$ ns.

Taking advantage of the constraints imposed on $k_{\mathrm{fs}}$ and $\tau_{\mathrm{w}}$, we may obtain several sets of parameters $\left(P_{\mathrm{s}}, S_{\mathrm{c}}, k_{\mathrm{fs}}\right.$, and $\left.\tau_{\mathrm{w}}\right)$ which have good fit to the SQ Lorentzian line width within the experimental error. The constrained nonlinear optimization package of MATLAB 5.1 (The Math Works Inc., MA), which is based on the sequential quadratic programming algorithm, was used to fit the full widths at half-height and one-third height of SQ spectra at two fields. Further screening for the good sets may be done by comparing the DQF spectra at creation time $\tau$ $=400 \mu$ s with regard to the three criteria described below for comparing DAY and USY systems: (1) The intensity ratio of central peak to the largest wiggling peak. The result is $P_{\mathrm{s}}$ sensitive. (2) The intensity ratio of the largest wiggling peak to the second largest wiggling peak. The result is $S_{\mathrm{c}}$ sensitive. (3) The intensity ratio of central peak to the largest negative peak. The result is $k_{\mathrm{fs}}$ sensitive. As compared to the NaY sysem, the experimental DQF spectra shows a simple structure. The following fitting criteria are used instead: (1) Separation of the negative doublet and the line width at half-height of DQF spectra with $\tau=40 \mu$ s are invoked to evaluate $P_{\mathrm{s}}$ and $S_{\mathrm{c}}$. (2) The intensity ratio of the central peak to the negative peak at $\tau=$ $400 \mu \mathrm{s}$ is invoked to evaluate $k_{\mathrm{fs}}$. Following the above comparisons, we may exclude most of the inappropriate sets of parameters. Then we use the remaining sets of parameters to simulate the DQF spectra with other creation times and make further screening of $P_{\mathrm{f}}, S_{\mathrm{c}}$, and $k_{\mathrm{fs}}$ set. According to the relative intensity of the central peak in the DQF spectra at various creation times, we may then choose the best parameter set with the refined $\tau_{\mathrm{w}}$. It is found that there exits no other distinct set of parameters to fit the DQF spectra equally well. It was found that the $T_{1}$ is not sensitive to the estimated value of $P_{\mathrm{s}}, S_{\mathrm{c}}$, and $k_{\mathrm{fs}}$ (or $k_{\mathrm{sf}}$ ), but very sensitive to the choice of $\tau_{\mathrm{i}}$, which is $1-2$ orders smaller than $\tau_{\mathrm{w}}$. Finally, we use the parameter set to calculate the $T_{1}$ values at 76.78 and $46.07 \mathrm{MHz}$ in order to obtain an unambiguous $\tau_{\mathrm{i}}$ value.

Results of DQF Spectra and $\boldsymbol{T}_{\mathbf{1}}$ Values. Figures 4 and 5 show the experimental DQF spectra together with their corresponding simulations, for low-loading and high-loading samples, respectively, over a range of temperatures at $76.78 \mathrm{MHz} .{ }^{2} \mathrm{H}$ longitudinal relaxation time measurements were conducted over the same temperature range as for the DQF and line-width measurements. The experimental and calculated $T_{1}$ results obtained at the two resonance frequencies for both low- and high-loading samples are listed in Tables 1 and 2, respectively. Except for the $T_{1}$ data for the DAY sample at $76.78 \mathrm{MHz}$ and $210 \mathrm{~K}$, the consistency between experimental and calculated results in these two fields is excellent.

\section{Discussion}

The faujasite zeolites possess different adsorption sites in the supercage. Adsorption of benzene has been studied by infrared spectroscopy over NaY, DAY, and USY. ${ }^{3,5}$ In NaY, it is wellknown that two pairs of bands correspond to the $v_{5}+v_{17}$ and $v_{10}+v_{17}$ vibrations of liquid benzene at 1960 and $1815 \mathrm{~cm}^{-1}$, and the consequent shift to higher wavenumbers and split into two pairs of bands is related to benzene adsorption on $\mathrm{S}_{\text {II }}$ sites and on oxygen atoms of 12 ring windows (or $\mathrm{W}$ sites), respectively. ${ }^{4}$ The high-wavenumber peak assigned to benzene adsorbed on the $\mathrm{W}$ site grows with a benzene loading higher than 2-3 molecules per supercage. For lower-loading samples, benzene gives only one pair of $\mathrm{CH}$ bands assigned to benzene interacting with the $\mathrm{Na}$ cations at $\mathrm{S}_{\mathrm{II}}$ sites. This agrees with the result of X-ray powder diffraction ${ }^{35}$ and MQMAS evaluation of ${ }^{23} \mathrm{Na}$ isotropic shift and second-order quadrupolar effect. ${ }^{17}$ Molecular mechanics simulation also confirms such benzene sites on different positions in NaY. ${ }^{35}$ Both of the above results show that adsorption at $\mathrm{W}$ sites is weaker than that occurring with benzene at $\mathrm{I}_{\mathrm{S}}$ sites. As for benzene adsorbed on DAY and USY, the interaction of the benzene $\pi$-electron cloud with the hydroxyl aluminum sites results in adsorption in such a system, while no significant adsorption interaction of benzene on the 
TABLE 3: Parameters Used in the Fitting of $T_{1}$, Line Width, and Double Quantum Filtered Spectra for Low-Loading Samples ${ }^{a}$

\begin{tabular}{ccccccc}
\hline zeolite & $\begin{array}{c}\text { temp } \\
(\mathrm{K})\end{array}$ & $P_{\mathrm{s}}$ & $S_{\mathrm{c}}$ & $\tau_{\mathrm{i}}(\mathrm{ns})$ & $\tau_{\mathrm{w}}(\mathrm{ns})$ & $\begin{array}{c}k_{\mathrm{fs}} \times \\
10^{-2}\left(\mathrm{~s}^{-1}\right)\end{array}$ \\
\hline NaY & 290 & 0.90 & 0.04 & $65 \pm 3$ & $350 \pm 20$ & 129 \\
& 310 & 0.87 & 0.02 & $42 \pm 3$ & $220 \pm 30$ & 87.0 \\
& 330 & 0.75 & 0.02 & $23 \pm 3$ & $120 \pm 10$ & 68.2 \\
& 350 & 0.75 & 0.02 & $20 \pm 2$ & $60 \pm 5$ & 62.5 \\
DAY & 210 & 0.75 & 0.9 & $0.15 \pm 0.02$ & $50 \pm 4$ & 50.0 \\
& 250 & 0.60 & 0.5 & $0.13 \pm 0.05$ & $20 \pm 2$ & 24.0 \\
& 290 & 0.52 & 0.3 & $0.11 \pm 0.05$ & $13 \pm 1$ & 10.4 \\
USY $^{b}$ & 330 & 0.43 & 0.3 & $0.10 \pm 0.03$ & $10 \pm 1$ & 7.82 \\
& 210 & 0.92 & 1.1 & $0.11 \pm 0.01$ & $70 \pm 5$ & 46.0 \\
& 250 & 0.80 & 1.4 & $0.08 \pm 0.01$ & $16 \pm 2$ & 23.5 \\
& 290 & 0.68 & 1.7 & $0.06 \pm 0.02$ & $7.5 \pm 1$ & 8.5 \\
& 330 & 0.60 & 5.0 & $0.04 \pm 0.03$ & $4.5 \pm 3$ & 4.6
\end{tabular}

${ }^{a} 10 \%$ error is expected in $k_{\mathrm{fs}}, 5 \%$ error in $P_{\mathrm{s}}$, and $20 \%$ error in $S_{\mathrm{c}}$. ${ }^{b}$ This result has been shown in ref 16 .

W sites has been detected. Auerbach et al. used a kinetic Monte Carlo method to calculate site-to-site hopping activation energies $^{11-13}$ and found several kinds of site-to-site hopping motions between 200 and $300 \mathrm{~K}$, e.g., $\mathrm{S}_{\mathrm{II}} \leftrightarrow \mathrm{S}_{\mathrm{II}}, \mathrm{S}_{\mathrm{II}} \leftrightarrow \mathrm{W}, \mathrm{W}$ $\leftrightarrow \mathrm{S}_{\mathrm{II}}$, and $\mathrm{W} \leftrightarrow \mathrm{W}$ within the rate ranging from $10^{4}$ to $10^{8} \mathrm{~s}^{-1}$ for benzene in $\mathrm{NaY}$. This may set a time scale for site hopping and also for the concomitant small-angle reorientation. ${ }^{15}$ It allows an efficient average for the processes for NaY system. The same condition is assumed to be available in DAY system.

Furthermore, the application of modified cone model with site-to-site hopping process in the present work is qualitatively similar to the model of benzene orientation randomization (BOR) used by Auerbach and Meitu. ${ }^{13}$ In the early time of orientation correlation function, the orientation randomization in their model is associated with the site-to-site hopping process. The long-time behavior of orientational correlation function may be correlated to the $\tau_{\mathrm{w}}$ process of present model since the wobbling motion is induced by the benzene mobility in the cage. The long-time BOR rate gives a correlation time $500 \mathrm{~ns}$ at 300 K. It agrees well with $\tau_{\mathrm{w}} \sim 300 \mathrm{~ns}$ for our NaY system at this temperature. The long-time BOR rate is controlled by the rate of cage-to-cage motions. This suggests a new approach from NMR relaxations to estimate self diffusion coefficients in zeolites. Although the wobbling motion described here and the small-angle reorientation found by Isfort et al. both result in small-angle reorientation for benzene molecules on adsorption sites, the former is related to $\mathrm{S}_{\mathrm{II}^{-}}-\mathrm{W}$ hopping while the later is associated with $\mathrm{S}_{\mathrm{II}}-\mathrm{S}_{\mathrm{II}}$ hopping. Since there are differences in time scale and the physical origin of the above two processes, one may decouple these two processes. Even without intracage tetrahedral jumps between adjacent sites, e.g. in the USY samples, the wobbling motion still exists and it is associated with intercage diffusion.

Low-Loading Samples. In accordance with the result of the fitting shown in Table 3, smaller magnitudes of $S_{\mathrm{c}}$ may be expected in the NaY or DAY systems that contrast with the $S_{\mathrm{c}}$ value observed in the USY system. Therefore, one may expect a small correlated order parameter $S_{\mathrm{c}}$ (or residual interaction $\omega_{\mathrm{c}}$ ) for a faujasite with more symmetrically arranged multiadsorption sites in the same supercage. Owing to these differences, the DQF spectra recorded in Figure 4 for benzene adsorbed in DAY frameworks show less wiggling in the shoulder of the spectral peak than in that for USY published previously. ${ }^{23}$ It is also distinctly different from those acquired for the benzene/ $\mathrm{NaY}$ system which gives the smallest $S_{\mathrm{c}}$ value. According to the line width listed in Table 1, due to a higher $\mathrm{Si} / \mathrm{Al}$ ratio,
TABLE 4: Parameters Used in the Fitting of $T_{1}$, Line Width, and Double Quantum Filtered Spectra for High-Loading Samples ${ }^{a}$

\begin{tabular}{|c|c|c|c|c|c|c|}
\hline zeolite & $\begin{array}{l}\text { temp } \\
(\mathrm{K})\end{array}$ & $P_{\mathrm{s}}$ & $S_{\mathrm{c}}$ & $\tau_{\mathrm{i}}(\mathrm{ns})$ & $\tau_{\mathrm{w}}(\mathrm{ns})$ & $k_{\mathrm{fs}} \times 10^{-2}\left(\mathrm{~s}^{-1}\right)$ \\
\hline \multirow[t]{3}{*}{$\mathrm{NaY}$} & 290 & 0.90 & 0.04 & $112 \pm 5$ & $300 \pm 20$ & 300 \\
\hline & 310 & 0.75 & 0.02 & $75 \pm 5$ & $210 \pm 25$ & 136 \\
\hline & 330 & 0.60 & 0.02 & $56 \pm 3$ & $110 \pm 15$ & 120 \\
\hline \multirow[t]{3}{*}{ DAY } & 210 & 0.37 & 0.9 & $0.39 \pm 0.1$ & $10 \pm 2$ & 18.5 \\
\hline & 250 & 0.30 & 0.3 & $0.14 \pm 0.1$ & $10 \pm 3$ & 8.57 \\
\hline & 290 & 0.28 & 0.3 & $0.10 \pm 0.05$ & $10 \pm 3$ & 7.00 \\
\hline \multirow[t]{3}{*}{ USY } & 210 & 0.28 & 0.2 & $0.40 \pm 0.01$ & $40 \pm 5$ & 9.33 \\
\hline & 250 & 0.27 & 0.3 & $0.20 \pm 0.01$ & $14 \pm 2$ & 5.40 \\
\hline & 290 & 0.25 & 0.3 & $0.09 \pm 0.03$ & $8 \pm 3$ & 5.00 \\
\hline
\end{tabular}

${ }^{a} 10 \%$ error is expected in $k_{\mathrm{fs}}, 5 \%$ error in $P_{\mathrm{s}}$, and $20 \%$ error in $S_{\mathrm{c}}$.

which has fewer cations, and weaker hydroxyl aluminum sites are expected in the DAY and USY systems, benzene becomes more mobile within these systems. ${ }^{22}$ The temperature dependence of the simulation parameters is shown in Table 3 for lowloading samples. As expected for a thermally activated process, $P_{\mathrm{s}}$ and $k_{\mathrm{fs}}$ decrease with the increase of temperature. From the Arrhenius analysis for the data shown in Table 3, the activation energy of wobbling motion and in-plane rotation for the $\mathrm{NaY}$ sample are much larger than those for the DAY and USY samples. Furthermore, the magnitudes of both $\tau_{\mathrm{w}}$ and $\tau_{\mathrm{i}}$ are in the order NaY > DAY > USY. They all indicate that the mobility of benzene is strongly correlated with the number of adsorption sites and the strength of adsorption. The result agrees well with the fact that the enthalpy of adsorption for a benzene$\mathrm{NaY}$ system is about $5 \mathrm{kcal} / \mathrm{mol}$ cations per unit cell larger than that for a benzene-dealuminated Y system. ${ }^{36}$

It is expected that only $\mathrm{NaY}$ and DAY may possess multiple adsorption sites available for benzene. The process of site-tosite hopping may reduce the net strength of residual quadrupolar interaction and therefore the decrease of $S_{\mathrm{c}}$ in NaY and DAY systems with increasing temperature. However, in contrast, the $S_{\mathrm{c}}$ value in the USY system increases with increasing temperature. From the physical significance of $\tau_{\mathrm{j}}$ and $D$ in the jump model described in the Appendix, their temperature dependency is in the opposite direction. In multiple site adsorption such as in $\mathrm{NaY}$, as the temperature rises, the decrease of $\tau_{\mathrm{j}}$ dominating the effect of increasing $D$ gives a decrease in $S_{\mathrm{c}}$, as shown in Table 3 (also see Table 4). For the USY system, since there is a lack of site-to-site hopping, the diffusion process is the dominant contributor to the increase of $S_{\mathrm{c}}$.

High-Loading Samples. It is found that benzene adsorbed at $\mathrm{S}_{\mathrm{II}}$ sites within $\mathrm{NaY}$ zeolite gives an average displacement from the $\mathrm{Na}^{+}$ion of about $3 \AA .{ }^{37-40}$ From ${ }^{23} \mathrm{Na}$ MQMAS study, more symmetrical surrounding of quadrupolar interaction is found in $\mathrm{NaY}$ with a increasing of benzene loading. ${ }^{17}$ Besides, for a loading of three benzene molecules per supercage, studied by ${ }^{1} \mathrm{H}$ MQ-NMR, a much shorter MQ coherence time or a much faster spin network growth rate is observed in the NaY system. ${ }^{16}$ Thus, in high-loading samples, occupation of different $\mathrm{Na}^{+} \mathrm{S}_{\mathrm{II}}$ sites by benzene molecules in the same supercages would generate barriers for benzene rotation. This supports our observation that a slower internal rotation rate was found. For benzene adsorbed on hydroxyl aluminum sites of DAY and USY, there are about 12 and 4.8 hydroxyl aluminum sites per unit cell of faujasite. However, one cannot be sure whether the locations of the hydroxyls are in the supercage or in the sodalite cage. There are less than 1.5 and 0.6 hydroxyl aluminum sites per supercage in DAY and USY, respectively. Due to a small number of benzene sites and a weaker adsorption interaction in these system, the intermolecular distance between benzene 
molecules may not be as close as in NaY system. Also, as compared with those in low-loading systems (cf. Table 3 and 4), one may infer that the rotation barriers resulted from high benzene loading slow down the in-plane rotation appreciably in all these faujasite systems. However, in DAY and USY systems the wobbling motion correlation time $\tau_{\mathrm{w}}$ of high-loading samples is significantly smaller than that of low-loading samples, whereas negligible differences are found between low- and highloading $\mathrm{NaY}$ samples. This may be due to a stronger strength of adsorption and more $\mathrm{Na}^{+}$sites in the latter system. With increasing loading in DAY and USY, additional benzene molecules are hardly affected by the interaction with weak adsorption sites. Thus the loading effects account for the smaller values of $\tau_{\mathrm{w}}$ and $\mathrm{k}_{\mathrm{fs}}$ found in these systems.

In Tables 3 and 4 , except for the $\tau_{\mathrm{i}}$ and $\mathrm{k}_{\mathrm{fs}}$ processes, no loading effect is found for the other fitting parameters in the $\mathrm{NaY}$ system. The study of powder neutron diffraction for $\mathrm{NaY}$ indicates the displacement, by about $0.09 \AA$, of $\mathrm{Na}$ in $\mathrm{S}_{\mathrm{II}}$ sites along the cube diagonal toward the center of the supercage. This reflects the adsorption of benzene on $\mathrm{S}_{\text {II }}$ sites becoming a little more disordered with the increasing loading. ${ }^{17,37}$ Therefore, only negligible effects on $S_{\mathrm{c}}$ are found in high-loading samples. However, in DAY and USY the magnitude of $P_{\mathrm{S}}$ is dramatically reduced with loading. Unlike the case of $\mathrm{NaY}$, there are less adsorption sites per supercage in DAY and USY. Therefore, the chance to find appropriate sites situated in the same supercages simultaneously to accommodate additional benzene molecules is relatively rare in DAY and USY systems. The loading effect leads to a small $P_{\mathrm{s}}$ found in these systems.

\section{Conclusion}

This work presents more detailed results regarding the comparison between different faujasites and the loading effect of the benzene molecule in the supercage. For all samples, the magnitudes of $\tau_{\mathrm{w}}$ and $\tau_{\mathrm{i}}$ are strongly correlated with the strength of adsorption and the free volume available for reorientation. $S_{c}$ values can be used to differentiate the nature of residual quadrupole interaction remaining, after averaging over the distribution of adsorption sites, from the process of site-to-site hopping. A slower internal rotation is found in $\mathrm{NaY}$ with increasing loading. It is consistent with the facts that the displacement of benzene from $S_{\text {II }}$ site is toward the center of supercage, ${ }^{37-40}$ and then a closer seperation of benzene molecules in NaY supercages is expected. DQF spectral analysis in conjunction with the usual relaxation studies may be a better combination of NMR relaxation methods for characterizing the dynamics and adsorption interaction in the zeolite system. This study forms part of a continuing series of studies of NMR methods for the investigation of diffusion and adsorption in porous materials.

Acknowledgment. This work was supported by the National Science Council of the Republic of China under Grant No. NSC 86-2113-M-002-025 and No. NSC 87-2113-M-002-039. We also thank Dr. Hong-Ping Lin for his assistance in $\mathrm{N}_{2}$ adsorptiondesorption isotherm measurement.

\section{Appendix}

In our approach to the exchange adsorption process, it may be unnecessary to specify the site interaction precisely. Considering the exchange process to be of diffusive origin may be sufficient. The motion of adsorbate may be described by the diffusion equation in the space specified by the adsorption coordinate $S$, e.g.

$$
\frac{\partial P(S, t)}{\partial t}=D \frac{\partial^{2} P(S, t)}{\partial S^{2}}
$$

where $D$ is the "diffusion" coefficient in units of s ${ }^{-1}$. Here, the probability after time $t$ that it will be at site $S$ in $\mathrm{d} S$ relative to the initial site at $S=0$ is given by the solution of eq A1.

$$
P(S, t)=\frac{1}{\sqrt{\pi D t}} \exp \left(-S^{2} /(4 D t)\right)
$$

The adsorbate molecules are considered to spend most of the time, characterized by $\tau_{j}$, residing in a given site with $S \neq 0$ before moving to the subsequent site. Similar to the treatment in Torrey theory ${ }^{41}$ for jump diffusion, the time intervals $t$ will not be constant, but will be governed by a probability distribution of an exponential form. Thus, in the present case, the probability density $f(S)$ along the adsorption coordinate may be obtained by multiplying eq A1 by $\exp \left(-t / \tau_{j}\right) \mathrm{d} t / \tau_{j}$ and then integrating the resulting expression over $t$. Hence

$$
\begin{aligned}
f(S) & =\int_{0}^{\infty} P(s, t) \exp \left(-t / \tau_{j}\right) \frac{\mathrm{d} t}{\tau_{j}} \\
& =\frac{1}{\sqrt{D \tau_{j}}} \exp \left(-S / \sqrt{D \tau_{j}}\right)
\end{aligned}
$$

The probability density follows the exponential distribution which has been adopted in eq 15, although the normalization factor is modified for the present case with boundary conditions set at $S=0$ and $S=1$. One may readily identify the factor $S_{\mathrm{c}}$ in the exponent of eq 15 as $\left(D \tau_{j}\right)^{1 / 2}$. $\left(D \tau_{j}\right)^{1 / 2}$ may be considered to be a characteristic jump distance in $S$ space. Beyond this distance the probability $f(S)$ drops off.

\section{References and Notes}

(1) Lechert, H. Catal. Rev. Sci. Eng. 1976, 14, 1.

(2) Eckman, R.; Vega, A. J. J. Am. Chem. Soc. 1983, 105, 4841.

(3) Barthomeuf, D. Catal. Rev. 1996, 38, 521, and pertinent references therein.

(4) de Mallmann, A.; Barthomeuf, D. J. Chem. Soc., Chem. Commun. 1989, 129.

(5) Su, B.-L. Stud. Surf. Sci. Catal. 1995, 97, 303.

(6) Datka, J.; Sulikowski, B.; Gil, B. J. Phys. Chem. 1996, 100, 11242.

(7) Pfeifer, H. NMR-Basic Principles and Progress; Springer; Berlin,

1972; Vol. 7.

(8) Pfeifer, H. Phys. Rep. 1976, 26, 293 (Phys. Lett., Sect. C).

(9) Zibrowius, B.; Caro, J.; Pfeifer, H. J. Chem. Soc., Faraday Trans. 1 1988, 84, 2347.

(10) Demontis, P.; Yashonath, S.; Klein, M. C. J. Phys. Chem. 1989, 93,5016 .

(11) Auerbach, S. M.; Henson, N. J.; Cheetham, A. K.; Metiu, H. I. J. Phys. Chem. 1995, 99, 10600.

(12) Auerbach, S. M.; Metiu, H. I. J. Chem. Phys. 1996, 105, 3753.

(13) Auerbach, S. M.; Meitu, H. I. J. Chem. Phys. 1997, 106, 2893.

(14) Schaefer, D. J.; Favre, D. E.; Wilhelm, M.; Weigel, S. J.; Chmelka, B. F. J. Am. Chem. Soc. 1997, 119, 9252.

(15) Isfort, O.; Boddenberg, B.; Fujara, F.; Grosse, R. Chem. Phys. Lett. 1998, 288,71

(16) Pearson, J. F.; Chmelka, B. F.; Shykind, D. N.; Pines, A. J. Phys. Chem. 1992, 96, 8517 .

(17) Hu, K.-N.; Hwang, L.-P. Solid State Nucl. Magn. Reson. 1998, 12, 211

(18) Eliav, U.; Navon, G. J. Magn. Reson. 1994, B 103, 19

(19) Eliav, U.; Shinar, H.; Navon, G. J. Magn. Reson. 1992, 98, 223.

(20) Sharf, Y.; Eliav, U.; Shinar, H.; Navon, G. J. Magn. Reson. 1995, B107, 60 .

(21) Bull, L. M.; Henson, N. J.; Cheetham, A. K.; Newsam, J. M.; Heyes, S. J. J. Phys. Chem. 1993, 97, 11776.

(22) Sousa Goncalves, J. A.; Portsmouth, R. L.; Alexander, P.; Gladden L. F. J. Phys. Chem. 1995, 99, 3317.

(23) Chen, Y.-H.; Chang, W.-T.; Jiang, P.-C.; Hwang, L.-P. Microporous Mesoporous Mater. 1998, 21, 651. There are misprints in the exchange 
rate term. The correct one is shown in eq 5 in the present work. Also the expressions of $S$ and $\left\langle D_{00}^{(4)}\right\rangle_{\mathrm{s}}$ should be corrected as obtained $S=1 / 2 \cos \beta(1$ $+\cos \beta)$ and $\left\langle D_{00}^{(4)}\right\rangle_{\mathrm{s}}=(S / 8)\left(28 S-7(1+8 S)^{1 / 2}+1\right)$, respectively.

(24) Boddenberg, B.; Burmeister, R. Zeolite 1988, 8, 488.

(25) Boddenberg, B.; Beerwerth B. J. Phys. Chem. 1989, 93, 1440.

(26) Blum, K. Density Matrix Theory and Applications Plenum: New York, 1981. The component of the irreducible tensor sets (state multiple) presented in this reference is denoted by $T_{\mathrm{Kq}}$ which is the same as $\sigma_{\mathrm{Kq}}$ defined in this work.

(27) Ernst, R. R.; Bodenhausen, G.; Wokan, A. Principles of Nuclear Magnetic Resonance in One and Two Dimensions; Clarendon: Oxford, UK, 1987.

(28) Brink, D. M.; Satchler, G. R. Angular Momentum; Oxford University Press: London, 1968.

(29) Price, W. S.; Ge, N.-H.; Hong, L.-Z.; Hwang, L.-P. J. Am. Chem. Soc. 1993, 115, 1095.

(30) Jacobsen, J. P.; Bildsoe, H. K.; Schaumburg, K. J. Magn. Reson. 1976, 23, 153. There is a misprint in eq 32 . Instead of $5 \sqrt{2} / 7$ as printed, the coefficient of $\left\langle D_{00}^{(2)}\right\rangle$ in $J_{2}\left(2 \omega_{\mathrm{r}}\right)$ should be corrected as $10 / 7$.
(31) Brainard, J. R.; Szabo, A. Biochemistry 1981, 20, 4618.

(32) Wong, T. C.; Wang, P.-L., Duh, D.-M.; Hwang, L.-P. J. Phys. Chem. 1989, 93, 1295.

(33) Millett, F. S.; Dailey, B. P. J. Chem. Phys. 1972, 56, 3249

(34) Lambert, J. B.; Niehuis R. J.; Keepers J. W. Angew. Chem., Int. Ed. Engl. 1981, 20, 487.

(35) Klein, H.; Kirschhock, C.; Fuess, H. J. Phys. Chem. 1994, 98 , 12345 .

(36) Barthomeauf, D.; Ha, B.-H. J. Chem. Soc., Faraday Trans. I 1973, $69,2158$.

(37) Fitch, A. N.; Jobic, H.; Renouprez, A. J. Phys. Chem. 1986, 90 , 1311.

(38) Lechert, H.; Wittern, K.-P. Ber. Bunsen-Ges. Phys. Chem. 1978, 82,1054 .

(39) Germanus, A.; Koager, J.; Pfeifer, H.; Samulevic, N. N.; Zdanov, S. P. Zeolites 1985, 5, 91.

(40) Hoffmann, W.-D. Z. Phys. Chem. 1976, 257, 315.

(41) Torrey, H. C. Phys. Rev. 1953, 92, 962. 